\title{
A multimodal data collection tool using REALbasic and Mac OS X
}

\author{
PETER J. MOLFESE, TERRI L. BONEBRIGHT, THERESA M. HERMAN, and CATHERINE A. ROE \\ DePauw University, Greencastle, Indiana
}

\begin{abstract}
This project uses REALbasic 3.5 in the Mac OS X environment for development of a configuration tool that builds a data collection procedure for investigating the effectiveness of sonified graphs. The advantage of using REALbasic with the Mac OS X system is that it provides rapid development of stimulus presentation, direct recording of data to files, and control over other procedural issues. The program can be made to run natively on the new Mac OS X system, older Mac OS systems, and Windows (98SE, ME, 2000 PRO). With modification, similar programs could be used to present any number of visual/auditory stimulus combinations, complete with questions for each stimulus.
\end{abstract}

In the past, there has been a good deal of research performed to determine how people comprehend visually graphed data (Attali \& Goldschmidt, 1996; Carpenter \& Shah, 1998; Carswell, 1992; Curcio, 1987; Fischer, 2000; Gobbo, 1994; Jones \& Careras, 1996; Kosslyn, 1989; Lee \& Gerber, 1999; Pinker, 1996; Tufte, 1983). In contrast, research on the effectiveness of sonified graphs is in its infancy. Sonified graphs have a sound component added to the displayed visual data, so that the $x$-axis is used for time and the $y$-axis corresponds to change in frequency. For example, a scatterplot with a strong linear trend and a slope of 1 would be sonified by representing each point on the graph by a single note that would result in an increase in pitch as each point of the graph is "played" from left to right.

There has been some prior work on sonified graphs, using simulated data sets with different types of graphs (Flowers, Buhman, \& Turnage, 1997; Flowers \& Hauer, 1992, 1995), whereas other researchers have focused on sonified graphs of complex data sets in research settings (Blattner, Greenberg, \& Kamegai, 1990; Lunney \& Morrison, 1990; Scaletti \& Craig, 1990). However, there has been little research to date in which the use of sound, in addition to visual displays, for real data sets that are used in educational settings has been investigated. Therefore, the present research program was developed to extend the research on visual graph comprehension and sonified graphs to the types of data students are exposed to during their academic coursework.

In order to collect the data for the sonified graphs project, we required a computerized data collection tool. This tool had to meet a series of requirements for the creation

The work reported here was supported by a Student/Faculty Summer Research grant from Faculty Development at DePauw University. The authors thank Walter K. Beagley and an anonymous reviewer for their helpful comments on an earlier version of the manuscript. Correspondence concerning this article or requests for the program should be directed to T. L. Bonebright, Department of Psychology, DePauw University, Greencastle, IN 46135 (e-mail: tbone@ depauw.edu). of an appropriate experimental procedure. The data collection program had to be able to present random orders of both auditory and visual stimuli by using sound and graphics files, to record different response types and reaction times, and to save data to files that would be easy to transport into Excel or SPSS. In addition, this program had to run seamlessly on multiple platforms (PC and Mac) with a variety of operating systems and have a user-friendly interface for the participants. Finally, the configuration program for each of the conditions of the experiment had to be easy to use by research assistants who are not programmers. With these goals as guidelines, REALbasic 3.5 in the new Apple Macintosh Operating System (OS) version X environment was chosen for the development of the configuration program, the randomization tool, and the data collection procedure.

\section{METHOD}

\section{Operating System and REALbasic}

The Mac OS X system has improved applications programming interfaces (APIs), which are the frameworks that programmers use to develop applications for an operating system. The improved APIs allow for easier networking, more stable applications, better audio performance, and the ability to write programs that respond at runtime to a user's actions. With the introduction of Mac OS X, support was also increased for Java and other objectoriented programming languages (OOLs).

One application suite that utilizes OOL is called REALbasic, an interface-oriented environment with a modified BASIC backend. REALbasic allows programmers to easily manipulate objects, such as windows, buttons, movies, and timers, in the operating system environment. Traditionally, a programmer would have to use complicated APIs, which are designed to give a programmer control over the operating system. REALbasic simplifies specific APIs to allow easier manipulation and programming of system-wide objects. Another reason REALbasic was se- 
lected over other OOL environments was the ease of portability; with a simple compiler change, a REALbasic application can be recompiled to run on Windows and Macintosh platforms. One of the chief factors for using REALbasic was that the programming language is extremely easy to learn, which means that making future modifications to the program will be possible for programmers with various levels of experience. ${ }^{1}$

\section{Program Suite}

Through the use of REALbasic and several tools built into the programming environment for creating windows, using buttons, and incorporating QuickTime movies, the researchers were able to produce a suite of programs that consists of three parts: the configuration tool, a randomizing tool, and the data collection application.

Configuration tool. Researchers use the configuration tool to develop stimulus sets for later presentation to participants. The configuration interface has dialog boxes for inputs for each trial, including a graph file as a JPEG file, a sound file in MP3 format, and the graph description along with four questions for the graph, which can be entered from a word-processing file by using cut and paste (see Figure 1). Once all the information is entered, the program outputs a text file for each graph that contains the graph name, sound name, questions, and graph description, as well as the date at which the trial was created.

Presently, the program is set up to follow a very rigid structure of stimulus presentation and response collection. Future versions of the configuration tool will allow for greater flexibility in timing and for stimulus variations.

Randomizing tool. The randomization tool has no interface and runs automatically before each participant begins the data collection procedure. The application simply changes the order in which the questions are presented, which controls for order effects.

Data collection application. The final component of the program suite is the data collection application, which

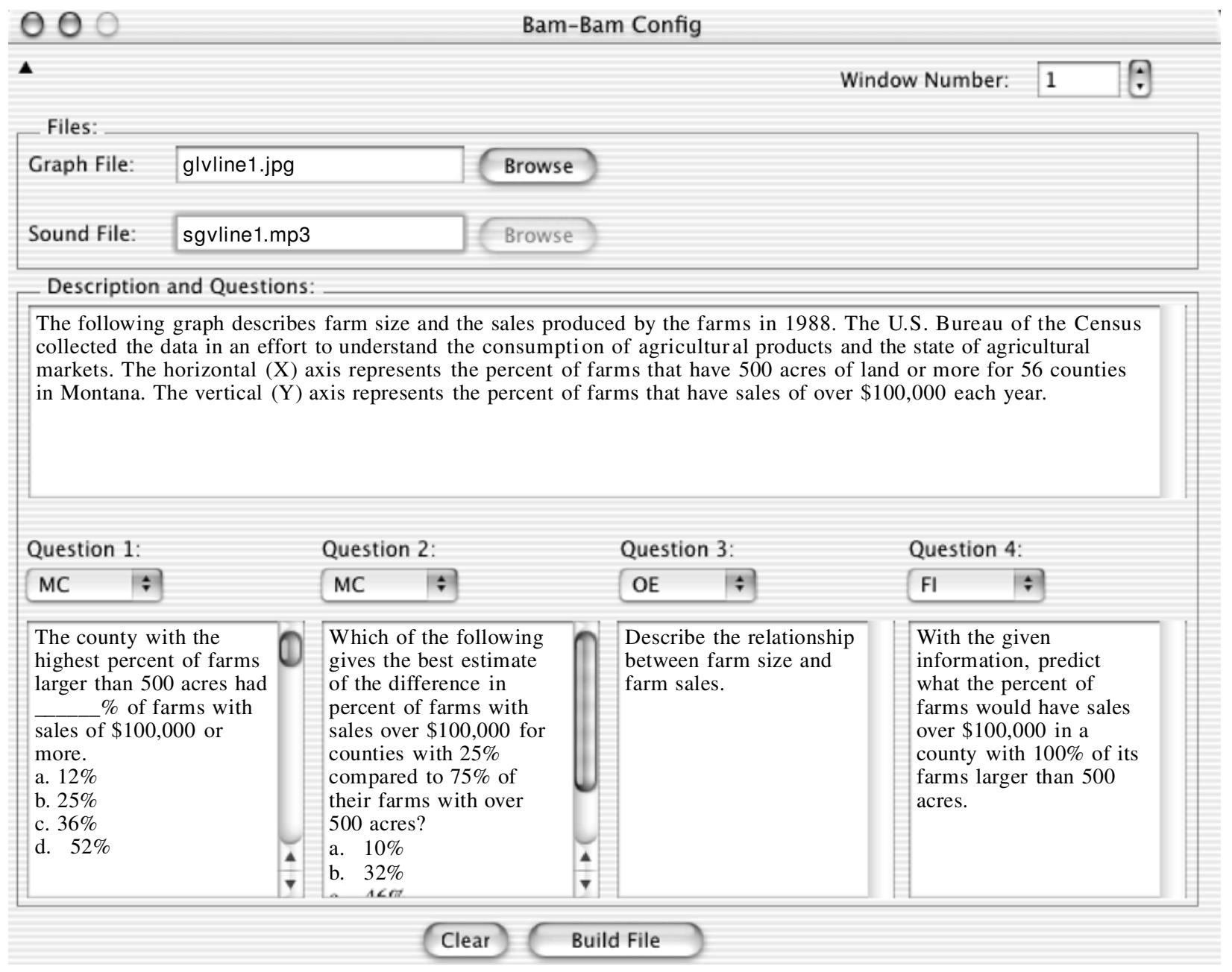

Figure 1. User interface for the configuration program. 


\section{Graph Description:}

Farm Sales in Relation to Farm Size for 56 Counties in Montana: The following graph describes farm size and the sales produced by the farms in 1988. The U.S. Bureau of the Census collected the data in an effort to understand the consumption of agricultural products and the state of agricultural markets. The horizontal $(X)$ axis represents the percent of farms that have 500 acres of land or more for 56 counties in Montana. The vertical $(Y)$ axis represents the percent of farms that have sales of over $\$ 100,000$ each year.

Figure 2. Example of a graph description screen from the data collection application.

is used to present the visual and auditory stimuli and to collect participant responses. The program begins with general instructions, including details about how the program works and how to exit the program at any time, should the participant decide not to continue the study. These general instructions are followed by a set of standard demographic questions. Subsequently, a set of four practice trials is presented to familiarize the participants with the

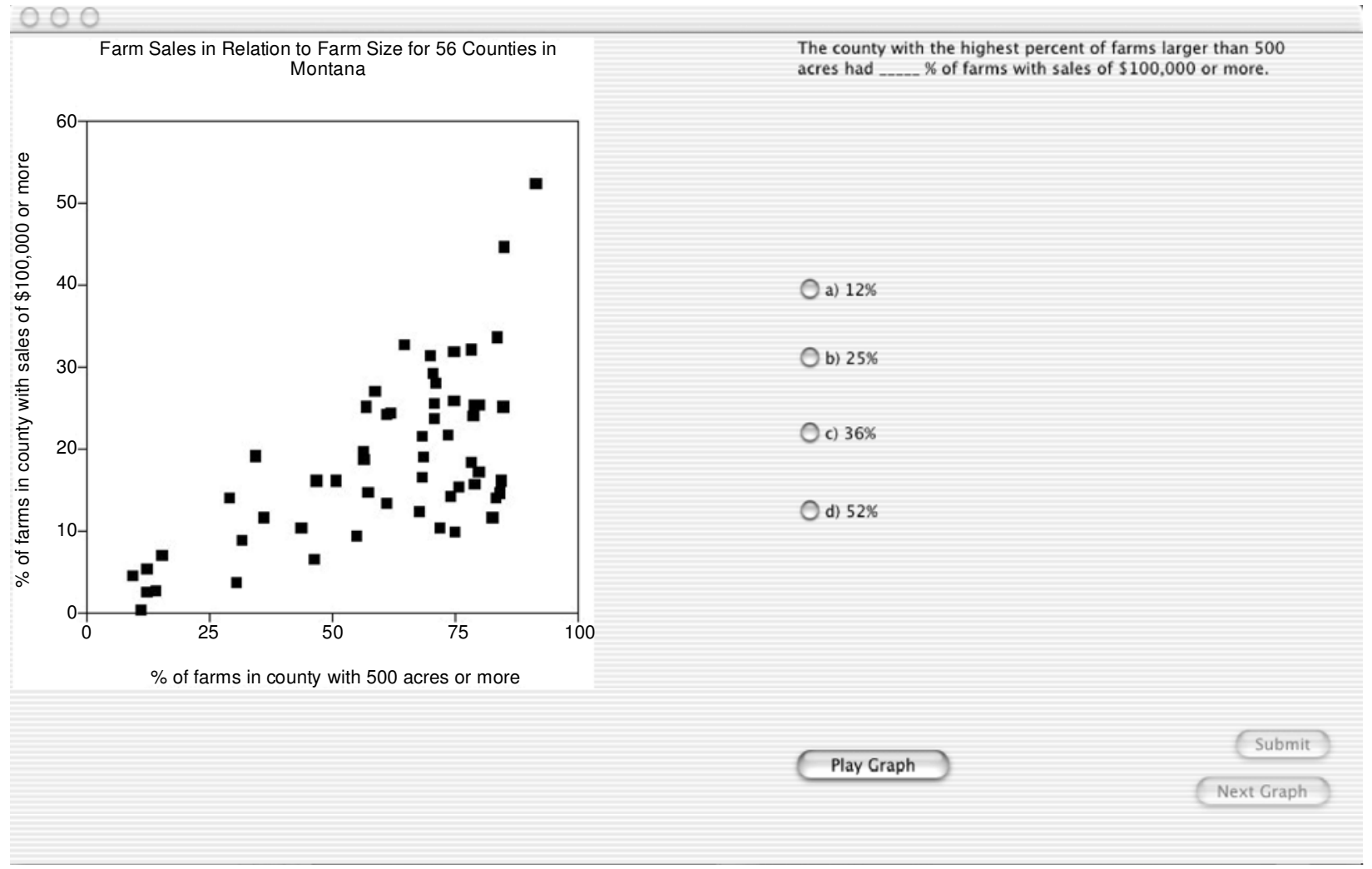

Figure 3. Example of a graph and a multiple-choice question from the data collection application. 
question types, the graph presentation, and other aspects of the procedure. The general format of these practice trials follows the same pattern as that described below in the section on test trials. Once the participants have completed the practice trials and asked any questions they may have, formal data collection begins.

For the test trials, each of the 28 graphs has four trialsone for each question type (specific, comparison, trend, and interpolation/extrapolation) -which are randomly ordered by the program. The participants answer the questions in multiple-choice, fill-in-the-blank, and open-ended formats. For each graph, the participant first reads the description of the data (see Figure 2). Then, on the following screen, the program presents either a line graph or a scatterplot along with the first question and plays the sonified version of the graph twice automatically through headphones (see Figure 3). While the participants are considering their responses, they may choose to listen to the sonification again by clicking on a button. The participants are instructed to determine the correct answer and to work as quickly as possible while still maintaining accuracy. Upon completing the first question, the participants are presented with the second question of four, and the sonification is again played twice. This process, in which graph description is followed by visual and auditory versions of the graph, is continued for all four questions associated with a specific graph and is repeated for all 28 graphs. Thus, the participants complete a total of 112 questions during the 1.5-h experimental session, which also includes two mandatory 2-min breaks to minimize fatigue.

The program records the participants' reaction times, their answers to the questions, and the number of times they played each sound. Data are placed in individual text files, for each participant, that can be read by Excel or SPSS.

Following the completion of the data collection application, the participants fill out a follow-up survey that asks about the difficulty of the tasks, the strategies used to understand the graphs, and the usefulness of the sounds during the trials. The experimental sessions are concluded with a full debriefing.

\section{CONCLUSION}

The present program is one example of the type of multimodal data collection tool that can be designed using REALbasic in the Mac OS X environment. The ease of learning the programming language, the program's flexibility to present random orders of different types of stimuli and to record a variety of response types, and the crossplatform compatibility make this a desirable program to add to a researcher's toolkit.

\section{REFERENCES}

Attali Y., \& Goldschmidt, C. (1996). The effects of component variables on performance in graph comprehension tests. Journal of Educational Measurement, 33, 93-105.

Blattner, M., Greenberg R. M., \& Kamegai, M. (1990, December). Listening to turbulence: An example of scientific audiolization. Paper presented at the ACM SIGCHI 90 Workshop on Multimedia \& Multimodal Interface Design, Seattle.

Carpenter, P. A., \& SHah, P. (1998). A model of perceptual and conceptual processes in graph comprehension. Journal of Experimental Psychology: Applied, 4, 75-100.

CARswell, C. M. (1992). Reading graphs: Interactions of processing requirements and stimulus structure. In B. Burns (Ed.), Percepts, concepts, \& categories (pp. 605-645). Amsterdam: Elsevier.

Curcio, F. R. (1987). Comprehension of mathematical relationships expressed in graphs. Journalfor Research in Mathematics in Education, 18, 382-393.

Fischer, M. H. (2000). Do irrelevant depth cues affect the comprehension of bar graphs? Applied Cognitive Psychology, 14, 151-162.

Flowers, J. H., Buhman, D. C., \& Turnage, K. D. (1997). Crossmodal equivalence of visual and auditory scatterplots for exploring bivariate data samples. Human Factors, 39, 341-351.

Flowers, J. H., \& Hauer, T. A. (1992). The ear's versus the eye's potential to assess characteristics of numeric data: Are we too visuocentric? Behavior Research Methods, Instruments, \& Computers, 24, 258-264.

Flowers, J. H., \& HAUer, T. A. (1995). Musical versus visual graphs: Cross-modal equivalence in perception of time series data. Human Factors, 37, 553-569.

Gовво, C. (1994). On children's understanding of an economic concept: The role of graphics in evaluation. In W. Schnotz \& R. W. Kulhavy (Eds.), Comprehension of graphics (pp. 225-249). Amsterdam: Elsevier.

Jones R. W., \& CAReras, I. E. (1996). The empirical investigation of factors affecting graphical visualization. Behavior Research Methods, Instruments, \& Computers, 28, 265-269.

Kosslyn, S. M. (1989). Understanding charts and graphs. Applied Cognitive Psychology, 3, 185-226.

LeE, J. C. K., \& Gerber, R. (1999). Hong Kong students' perceptions of graphs, charts and maps. Scandinavian Journal of EducationalResearch, 43, 19-40.

LunNey, D., \& Morrison, R. (1990). Auditory presentation of experimental data. Proceedings of the Society of Photooptical Instrumentation Engineers, 1259, 140-146.

Pinker, S. (1996). A theory of graph comprehension. In R. Freedle (Ed.), Artificial intelligence and the future of testing (pp. 73-126). Mahwah, NJ: Erlbaum.

ScaletTI, C., \& Craig, A.B. (1990). Using sound to extract meaning from complex data. Proceedings of the Society of Photooptical Instrumentation Engineers, 1259, 147-153.

TuFTE, E. R. (1983). The visual display of quantitative information. Cheshire, CT: Graphics Press.

\section{NOTE}

1. Information about REALbasic can be found by accessing the URL http://www.realsoftware.com. The current version of the program is 4.0, and even though the program is a commercial product, it is available at an academic discount.

(Manuscript received November 13, 2001; revision accepted for publication March 26, 2002.) 